\title{
A Least-Square Model to Estimate Historical Percentages of Itinerant General Aviation Operations by Aircraft Types and Flight Rules at an Airport
}

\author{
Tao Li ${ }^{1}$ and Antonio A. Trani ${ }^{2}$ \\ ${ }^{1}$ The Department of Electrical Engineering, University of Texas at Arlington, Arlington, TX 76019, USA \\ ${ }^{2}$ Air Transportation Systems Laboratory, The Charles E. Via, Jr. Department of Civil and Environmental Engineering, \\ Virginia Polytechnic Institute and State University (Virginia Tech), Blacksburg, VA 24061, USA \\ Correspondence should be addressed to Tao Li; tao81@vt.edu
}

Received 1 March 2017; Revised 11 May 2017; Accepted 4 June 2017; Published 25 July 2017

Academic Editor: Andrea D’Ariano

Copyright (C) 2017 Tao Li and Antonio A. Trani. This is an open access article distributed under the Creative Commons Attribution License, which permits unrestricted use, distribution, and reproduction in any medium, provided the original work is properly cited.

Historical airport-level General Aviation (GA) activity plays an important role in airport managerial and operational decisionmaking processes. We developed a model to estimate historical percentages of itinerant GA operations by four aircraft types and two flight rules. The model is formulated as a least-square optimization model and can be applied to both towered and nontowered airports. We applied the model to airports in the Terminal Area Forecast and compared model estimates with the observed statistics. This study provides a method that could be used in the decision-making processes that require detailed historical GA operations.

\section{Introduction}

General Aviation (GA) is the operation of civilian aircraft for purposes other than commercial passenger or cargo transport. It is an essential part of the air transportation system in the United States (US). The Federal Aviation Administration (FAA) created the National Plan of Integrated Airport Systems (NPIAS) [1], which identifies airports that are significant to national air transportation and thus eligible to receive federal grants. In the NPIAS, more than $80 \%$ of the airports primarily serve GA. For airports that mainly serve commercial flights, GA could also be of great interest since they share airport resources (e.g., runway and airspace) with commercial flights.

In the literature, the importance of historical airport-level GA activity has been well recognized as it is a key input in many airport managerial and operational decision-making processes [2,3], such as allocating funding, forecasting GA demand, conducting environmental analysis, preparing economic impact studies, justifying airport development projects, and evaluating the need for new airport facilities.
This paper is devoted to estimating historical airport-level GA operations so that those decision-making processes could be better supported.

The FAA provides the general public with three data sets that contain historical GA activities in the US. The first one is from the General Aviation and Part 135 Activity Survey (GA survey). The survey enables the FAA to monitor GA fleet so that the demand for the National Airspace System (NAS) can be met [4]. The data from the survey mainly has national-level historical GA activities. The second one is from the Operations Network (OPSNET), which contains the official air traffic and delay data [5] of the NAS. In particular, it has historical itinerant GA operations by Instrument Flight Rules (IFR) and Visual Flight Rules (VFR). However, the data does not cover nontowered airports, which serve the majority of GA activities by aircraft types and flight rules. The third one is from the Terminal Area Forecast (TAF) [6]. The TAF is prepared to assist the FAA in meeting its planning, budgeting, and staffing requirements. It is also an important basis and benchmark used by many state airport authorities and other 
aviation planners for airport planning and improvements $[7,8]$. The TAF contains historical GA operations for both towered and nontowered airports. However, the GA operations are aggregated into itinerant and local operations (aircraft operating in the traffic pattern or within sight of the tower, or aircraft known to be departing or arriving from flight in local practice areas, or aircraft executing practice instrument approaches at the airport [6]; the FAA reports all aircraft operations other than local operations as itinerant; in essence, itinerant operations are takeoffs and landings of aircraft going from one airport to another) and little other details are available.

Many nontowered airports also have their own efforts to collect historical air traffic data. Center for Transportation Research and Education [9], Muia [3], Muia [10], and Muia and Johnson [11] reviewed these efforts. Two categories of method are commonly used.

The first category is to deploy air traffic counters to count the operations year-round. The commonly used counters include pneumatic counters, inductance loop counters, acoustical counters, and visual/video counters. The costs, accuracy, and level of details of the traffic counts vary among counters. For example, pneumatic counters have relatively low accuracy and cannot distinguish between aircraft types. Visual/video and sound-level counters could offer relatively accurate counts but equipment and maintenance costs are relatively high. Their data extraction cost could also be high if outputs need to be further reviewed to determine the counts. In addition, most of the counters cannot distinguish flight operations by flight rules and flight purposes (i.e., GA and commercial).

The second category is to estimate air traffic operations. The estimation could be made by counseling airport personnel or applying statistical/optimization methods. Compared with methods in the first category, this type of method is relatively inexpensive and could be a good option for scenarios in which it is not cost-beneficial to use other collecting methods. In the survey conducted in Muia [10], counseling airport personnel is the most commonly used method among all the surveyed airports; however, the estimates could be of relatively low accuracy. When the actual counts are not available, the FAA recommends that appropriate statistical techniques be used. Statistical models [11-15] are developed using historical data to estimate historical airport-level GA operations. In the literature, similar statistical models are also developed to forecast airport-level GA operations (e.g., Ghobrial [16]; Dou et al. [17]; Li and Trani [18, 19]) using historical data. The statistical models mentioned above estimate GA activity by establishing a casual relationship between the activity and explanatory factors (e.g., social-demographical and economical factors). These models assume that GA activity is a result of the explanatory factors. Another type of estimation method utilizes statistical/optimization models to estimate air traffic operations with posterior observations, that is, observations as a result of air traffic operations. The posterior observations could be traffic sample collected by counters [2], fuel sales [20, 21], airport guest logs [10], or operations at towered airports $[11,22]$. These methods could produce relatively accurate and cost-beneficial estimates
[10, 23]. It is worthwhile to point out that models that utilize posterior observations have also been developed to estimate the historical demand in commercial air transportation (see, e.g., Li et al. [24]; Li and Baik [25]; Li [26]; Li et al. [27]; and $\mathrm{Li}$ [28]) and ground transportation (see, e.g., Maher [29]; Cascetta [30]; Bell [31]; Yang et al. [32]; Codina and Barceló [33]; Chootinan et al. [34]; Doblas and Benitez [35]; Nie et al. [36]; Lundgren and Peterson [37]; Chen et al. [38]).

In FAA's Advisory Circular (it provides guidelines for preparation of master plans for airports that range in size and function from small General Aviation to large commercial service facilities) [8], it is recommended that the data that identifies the category and class of aircraft and type of fuels and services that those aircraft use be collected. Historical data of GA operations by aircraft types and flight rules is one of the important inputs based on which many airport managerial and operational decisions would be made. For example, the number of operations under IFR is a key factor to determine the need for Instrument Landing Systems, instrument approach procedure development, control tower, and Air Traffic Control staff. Turbofan engine aircraft could be significantly different from piston engine aircraft in terms of, for instance, noise and emission level, separation standards, and service requirements (e.g., fuel types runway length and maintenance facilities). As a result, operations by aircraft type/aircraft mix would be needed to estimate capacity [39-41], optimize scheduling [42-46], and conduct environmental review $[47,48]$. However, to the best of the authors' knowledge, not all these data could be collected by traffic counters or estimated by existing statistical/optimization methods.

This study is aimed at filling in this gap. We developed an optimization model to estimate airport-level itinerant GA operations by four aircraft types (i.e., fixed-wing piston, fixed-wing turboprop, fixed-wing turbofan, and the rest of aircraft) and two flight rules (i.e., IFR and VFR) for both towered and nontowered airports. The classification of aircraft types and flight rules are based on these used in the GA survey. The model could be useful for the decision-making processes that require detailed historical GA operations. Since local GA operations are primarily performed by single-engine aircraft under VFR, we only focus on itinerant GA operations. The model is formulated as a least-square optimization problem, which considers statistics such as those in the FAA's data sets and those available at local airports. Instead of directly estimating the number, the model estimates the operations by aircraft type and flight rule in terms of percentage. The percentages could then be applied to the total number of itinerant GA operations to generate the number of operations by aircraft type and flight rule. In particular, the model can be applied to the itinerant GA operations in the TAF. For scenarios in which the total number or a separation between itinerant and local operations is not available, the data in the TAF or estimates from statistical models (e.g., Li and Trani [18]) could be used to provide the total number or a separation between itinerant and local operations. 


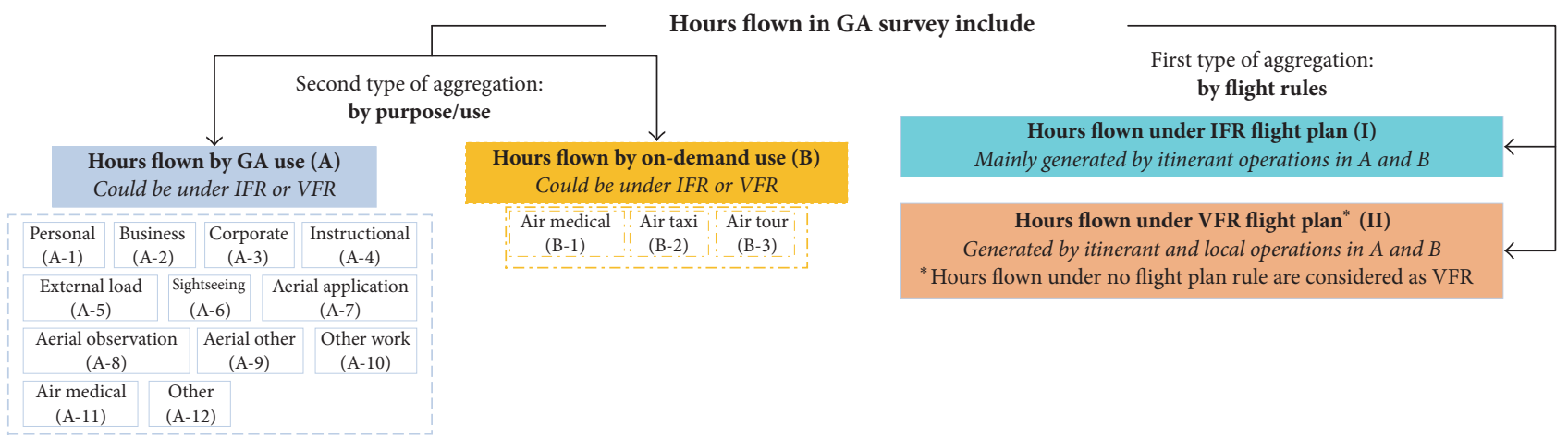

FIgURE 1: Two types of aggregation of hours flown in GA survey.

The rest of the paper is organized as follows: in Section 2, we introduce three data sets that are used in this study. In Section 3, we present the estimation model. In addition, for nontowered airports we present a logistic regression model to estimate a key coefficient in the objective of the estimation model. In Section 4, we apply the estimation model to airports in the TAF. We compare the estimation results with the observed ones. In Section 5, we summarize our study.

\section{Data Sets}

The General Aviation and Part 135 Activity Survey. This survey provides the FAA with a yearly snapshot of information on GA and on-demand part 135 aircraft activity [4]. The data from the survey is used in many areas such as identifying safety problems and forming the basis for research of GA issues (e.g., Federal Aviation Administration [49] and Li and Trani [50]).

The survey collects information on hours flown by use, flight conditions, and aircraft characteristics. For simplicity, we classify the aircraft in the survey into four aircraft types:

(1) Fixed-wing piston engine aircraft (piston, for short)

(2) Fixed-wing turboprop engine aircraft (turboprop)

(3) Fixed-wing turbofan engine aircraft (turbofan)

(4) The other aircraft, that is, the rest of the aircraft in the survey, such as rotorcraft, gliders, experimental, and amateur built.

There are three flight-rule plans:

(1) IFR Flight Plan. The flight plan is filed and the flight is flown according to rules and regulations established by the FAA to govern flight under conditions in which flight by outside visual reference is not safe. IFR flight depends upon flying by reference to instruments in the flight deck, and navigation is accomplished by reference to electronic signals.

(2) VFR Flight Plan. The flight plan is filed; however, the flight is flown solely by reference to outside visual cues (horizon, buildings, flora, etc.), which permit navigation, orientation, and separation from terrain and other traffic.

(3) No Flight Plan. That is, no flight plan is filed and the flight is flown under VFR.

Flight operations under the last two categories are both under VFR. Therefore, for simplicity, we call them VFR operations in this study. Itinerant GA operations could be performed under IFR or VFR while local GA operations are mostly performed under VFR.

In the survey data currently available to the general public, hours flown are aggregated in several ways. In this study, we used two types of aggregation. The first type is hours flown by flight plan. As shown in Figure 1, this aggregation includes hours flown by IFR flight plan and hours flown by VFR flight plan. Hours flown by IFR flight plan are primarily generated by itinerant GA operations. Hours flown by VFR flight plan could be generated by itinerant and local GA operations. The second type is hours flown by purpose/use. There are two subcategories in this aggregation. The first subcategory contains twelve flight purposes: personal, business, corporate, instructional, aerial application, aerial observation, aerial other, external load, other work, sightseeing, air medical, and other. The second subcategory includes three flight purposes: air taxi, air medical, and air tour. This subcategory is generally not considered as GA.

Flight Database in the Enhanced Traffic Management System (The ETMS Has Been Upgraded to Traffic Flow Management System [51]) (ETMS). The ETMS is the system used by the FAA to manage air traffic flow within the NAS. One of the core parts of the ETMS is the flight database [52]. Examples of flight records in the ETMS are shown in Table 1. The flight information in the flight database includes a flight's departure and arrival airports/times, aircraft information (e.g., aircraft engine type) used to perform the flight, and flight purpose (e.g., GA and scheduled commercial services).

Flight data available to our study is from 2004 to 2012. The data contains flight operations under IFR flight plan of 16 days from each year. The days are selected using a set of performance metrics and are representative of typical days 
TABLE 1: Examples of flight record in ETMS.

\begin{tabular}{lccccc}
\hline Date & Departure airport & Arrival airport & Aircraft type & Physical class & User class \\
\hline 20091004 & MRY & LIMT & DV20 & J & General aviation \\
20091005 & SYR & ACY & B732 & J & Unscheduled on-demand \\
20091005 & HOU & CUH & BE9L & T & Unscheduled on-demand \\
20091005 & HLN & $6 S 8$ & C340 & P & General aviation \\
20091005 & SUS & MDW & P28R & P & General aviation \\
20091005 & MIW & LNK & PC12 & T & General aviation \\
\hline
\end{tabular}

TABLE 2: Examples of airports operations in OPSNET 2010.

\begin{tabular}{lcccccccccc}
\hline \multirow{2}{*}{ Airport } & \multicolumn{4}{c}{} & \multicolumn{2}{c}{ IFR itinerant } & \multicolumn{3}{c}{ VFR itinerant } & \multicolumn{2}{c}{ Local } \\
& Carrier & Air taxi & GA $^{*}$ & Military & Carrier & Air taxi & GA $^{*}$ & Military & Civil & Military \\
\hline ABE & 5,050 & 16,354 & 14,480 & 436 & 1 & 644 & 16,758 & 195 & 41,565 & 275 \\
ILG & 41 & 3,640 & 13,587 & 1,512 & 3 & 237 & 17,291 & 1,327 & 22,667 & 6,032 \\
MMU & 8 & 10,200 & 20,054 & 45 & 0 & 1,389 & 48,729 & 174 & 37,987 & 184 \\
HFD & 17 & 2,500 & 3,141 & 21 & 0 & 1,721 & 31,422 & 30 & 32,124 & 54 \\
LGA & 218,843 & 141,114 & 4,663 & 230 & 3 & 121 & 2,293 & 79 & 0 & 0 \\
HOU & 99,337 & 23,626 & 46,814 & 258 & 7 & 11,842 & 18,290 & 1,582 & 340 & 0 \\
PDK & 9 & 12,366 & 55,053 & 264 & 4 & 1,153 & 54,237 & 182 & 37,640 & 41 \\
\hline
\end{tabular}

* This study primarily uses itinerant GA operations under IFR and VFR.

of flight operations in the NAS [53]. Similar sample data has been used in several other studies (see, e.g., $[17,19,54]$ ).

Operations Net (OPSNET). The FAA collects and analyzes the data in the OPSNET to make decisions on budgeting, forecasting, planning, and historical analysis [55]. Table 2 presents examples of flight operations at airports in OPSNET. The statistics may be collected either manually or using automated counting programs. However, the data is only available for FAA and FAA-contracted towers [56]. These airports consist of a relatively small portion of the airports that primarily serve GA.

\section{Model}

3.1. Notations and Formulation. For an airport, we have the following:

Decision variables:

$M_{P}$ : percentage of itinerant operations performed by piston aircraft

$M_{B}$ : percentage of itinerant operations performed by turboprop aircraft

$M_{J}$ : percentage of itinerant operations performed by turbofan aircraft

$M_{O}$ : percentage of itinerant operations performed by the other aircraft

$I_{P}$ : percentage of IFR itinerant operations performed by piston aircraft
$I_{B}$ : percentage of IFR itinerant operations performed by turboprop aircraft

$I_{J}$ : percentage of IFR itinerant operations performed by turbofan aircraft

$I_{O}$ : percentage of IFR itinerant operations performed by the other aircraft

Constants in the objective function:

$\bar{M}_{P}$ : target percentage of itinerant operations performed by piston aircraft

$\bar{M}_{B}$ : target percentage of itinerant operations performed by turboprop aircraft

$\bar{M}_{J}$ : target percentage of itinerant operations performed by turbofan aircraft

$\bar{M}_{\mathrm{O}}$ : target percentage of itinerant operations performed by the other aircraft

$\bar{I}_{P}$ : target percentage of IFR itinerant operations performed by piston aircraft

$\bar{I}_{B}$ : target percentage of IFR itinerant operations performed by turboprop aircraft

$\bar{I}_{J}$ : target percentage of IFR itinerant operations performed by turbofan aircraft

$\bar{I}_{O}$ : target percentage of IFR itinerant operations performed by the other aircraft

$\bar{M}_{\text {IFR }}$ : target percentage of IFR itinerant operations.

The least-square optimization model is formulated as follows: 
$\min f$

$$
\begin{aligned}
= & \alpha\left[\bar{M}_{\mathrm{IFR}}-\left(I_{P} \times M_{P}+I_{B} \times M_{B}+I_{J} \times M_{J}+I_{O} \times M_{O}\right)\right]^{2} \\
& +\beta\left[\left(I_{P}-\bar{I}_{P}\right)^{2}+\left(I_{B}-\bar{I}_{B}\right)^{2}+\left(I_{J}-\bar{I}_{J}\right)^{2}+\left(I_{O}-\bar{I}_{O}\right)^{2}\right]+\gamma\left[\left(M_{P}-\bar{M}_{P}\right)^{2}+\left(M_{B}-\bar{M}_{B}\right)^{2}+\left(M_{J}-\bar{M}_{J}\right)^{2}\right], \\
\text { s.t. } \quad & \theta_{P} \times M_{P}+\theta_{B} \times M_{B}+\theta_{J} \times M_{J}+\theta_{O} \times M_{O} \leq M, \\
& \pi_{P} \times M_{P}+\pi_{B} \times M_{B}+\pi_{J} \times M_{J}+\pi_{O} \times M_{O} \leq I, \\
& M_{P}+M_{B}+M_{J}+M_{O}=1, \\
& 0 \leq M_{P} \leq 1 \\
& 0 \leq M_{B} \leq 1 \\
0 \leq & M_{J} \leq 1 \\
0 \leq & M_{O} \leq 1 \\
0 \leq & I_{P} \leq 1 \\
0 \leq & I_{B} \leq 1 \\
0 \leq & I_{J} \leq 1 \\
0 \leq & I_{O} \leq 1 .
\end{aligned}
$$

The objective function (1) of the model is designed as a weighted least-square function. It has three terms. The first term can be derived by minimizing the difference between the target total number of IFR operations and the estimated one. That is,

$$
\begin{aligned}
& {\left[\bar{M}_{\mathrm{IFR}} \times T-\left(I_{P} \times M_{P} \times T+I_{B} \times M_{B} \times T+I_{J} \times M_{J}\right.\right.} \\
& \left.\left.\quad \times T+I_{O} \times M_{O} \times T\right)\right]^{2},
\end{aligned}
$$

where $T$ is the total number of operations (assumed to be a constant) at the airport, $\bar{M}_{\text {IFR }} \times T$ is the target total number of IFR operations, and $\left(I_{P} \times M_{P} \times T+I_{B} \times M_{B} \times T+I_{J} \times M_{J} \times\right.$ $\left.T+I_{O} \times M_{O} \times T\right)$ is the estimated total. Since $T$ is a constant, minimizing (7) is essentially the same as minimizing the first term. Another way to interpret the first term is that it requires estimated total percentage of IFR operations to be close to the target one. However, there may be multiple optimal solutions by minimizing only the first term. We introduce target values for the decision variables to keep the estimated percentages close to some reasonable ranges. The second term requires the estimated percentages of IFR operations by aircraft type be close to the target ones (i.e., $\bar{I}_{P}, \bar{I}_{B}, \bar{I}_{J}, \bar{I}_{O}$ ). The third term requires the estimated percentages of operations by aircraft type be close to the target ones (i.e., $\bar{M}_{P}, \bar{M}_{B}, \bar{M}_{J}, \bar{M}_{\mathrm{O}}$ ). The target percentages do not need to be error-free since the leastsquare function allows the estimated percentages to deviate from the target ones. They could be estimated, for example, by taking a sample of traffic data or consulting airport personnel.
The objective function is a weighted sum of the three terms, where $\alpha, \beta, \gamma$ are the corresponding weights. Each term is a sum squared difference between target percentages and estimated ones. It is necessary to point out that the first term plays an important role in the objective. This is because the optimal solution would always be the target percentages if only the second and third terms are used in the objective. In other words, it is the first term that drives the optimal solution away from the targeted ones. For towered airports, the airport-level operations count in the OPSNET could be used to calculate $\bar{M}_{\text {IFR }}$. However, for some nontowered airports, it may not always be cost-beneficial to calculate $\bar{M}_{\text {IFR }}$. Considering its importance, in the following section we developed an estimation method to calculate $\bar{M}_{\text {IFR }}$ for nontowered airports.

Constraints (2) and (3) are general inequality constraints to be determined by specifying the coefficients in the inequality. For example, if $\pi_{I}=-1, \pi_{P}=1$, and $I=0$ are used, then constraints (3) become $I_{P} \leq I_{J}$, which requires the percentage of piston operations under IFR to be no more than that of turbofan aircraft under IFR. We suggest that the coefficients be specified based on an airport's characteristics and situations. For some large hubs, constraints (2) could be specified as $\theta_{P}=1, \theta_{J}=-1$, and $M=0$, which is $M_{P} \leq M_{\mathrm{J}}$. It requires the percentage of turbofan operations be no less than that of piston. For many nontowered small GA airports, constraints (3) could be specified as $\pi_{P}=2$ and $I=1$, which is $I_{P} \leq 1-I_{P}$. It requires the percentage of piston operations under IFR to be no more than that of piston operations under VFR. Constraints (4) require that 
the percentages of operations by aircraft type sum up to one. Constraints (5) and (6) are imposed to guarantee that the decision variables (percentages) are between zero and one.

The model could also be formulated with aircraft classifications other than the one used in this study. For example, the aircraft classification in the TAF (i.e., single-engine aircraft, multiple-engine aircraft, jet-engine aircraft, helicopters, and others) could be used in the decision variables. Moreover, instead of percentage of operations, the decision variables could also be given in terms of number of operations (e.g., number of itinerant operations performed by piston aircraft and number of IFR itinerant operations performed by piston aircraft). However, target percentage of operations is easier to obtain than target number of operations in that the former could be estimated by using sample data while the latter may need complete data to estimate.

The optimization model can be solved using the Generalized Reduced Gradient (GRG) method. The method has been successfully implemented to solve industrial problems [57]. Similar to the Simplex method, the GRC method divides the decision variables into basic and nonbasic variables. The basic variables are substituted by the nonbasic variables using constraints. The gradient of the objective function is then determined as a function of nonbasic variables. The nonbasic variables are updated so that a decrease in the objective along the gradient is achieved. The basic variables are then updated by solving a system of equations using the new nonbasic variables. During this process, some basic variables may be replaced by nonbasic variables, for example, to maintain feasibility or avoid degeneracy [58]. There are several GRG codes available for distribution in the literature (see, e.g., Waren and Lasdon [59] and Pike [57]), and Microsoft Excel also has a GRG method available in its Solver.

\subsection{Estimation of the Target Percentage of IFR Itinerant GA} Operations for Nontowered Airports. In this section, an estimation method is developed for nontowered airports where it is not cost-beneficial to calculate $\bar{M}_{\mathrm{IFR}}$. The method calculates $\bar{M}_{\text {IFR }}$ for a nontowered airport by using the data at the towered airports that have some "similarities." Ford and Shirack [20] believe that operation data at towered airports may not be able to provide reliable estimates of operations for nontowered airports. This is partially because the towered ones have instrument approaches that make them accessible during inclement weather. However, a newer study done by Muia [10] suggests that this may not be the case anymore due to the use of global positioning system as well as the increasing number of nontowered airports with instrument approaches.

Three types of factors are considered to be used to evaluate the similarity between a towered airport and a nontowered one. They are (1) local social-economic and demographic factors such as population and income, (2) supply factors at an airport such as runway and service facilities, and (3) fleet mix of based aircraft at an airport.

However, we found that a nontowered airport and a towered airport could have similar local social-economic and demographic factors but significantly different percentages of IFR operations. For example, the Peachtree City-Falcon
Field airport is a nontowered GA airport located about 25 miles away from the Hartsfield-Jackson Atlanta International Airport, which is a large hub (based on percentage of total US passenger enplanements, the FAA groups primary commercial service airports into four categories: large, medium, and small hubs and nonhub airports [1]). Because of their close vicinity, the two airports could have similar local socialeconomic and demographic factors; however, the percentage of IFR operations at the two airports could be significantly different due to their different classifications. In addition, the control tower itself is an important supply factor that determines the percentage of IFR operations.

The fleet mix of based aircraft at an airport could be a significant determinant of the percentage of IFR operations at the airport. In the 2010 GA survey, the total number of hours flown under IFR accounted for about 65\% and $96 \%$ of the total hours flown by multiple-engine aircraft and jetengine aircraft. The corresponding number for the rest of the aircraft in the survey is about $14 \%$. It is reasonable to expect that airports with similar fleet mix of based aircraft could have similar percentages of IFR operations. Therefore, the fleet mix of the based aircraft at an airport is chosen as the factor to measure the similarity between towered airports and nontowered airports.

sThe idea of the estimation method is to first establish a relationship between the fleet mix of based aircraft and the percentage of IFR operations at towered airports. Then, this relationship is used to calculate $\bar{M}_{\text {IFR }}$ for a nontowered airport using the fleet mix of based aircraft at the airport. It should be pointed out that some of the operations at an airport are performed by nonbased aircraft. By using the fleet mix of based aircraft to estimate the percentage of IFR operations, we assume that the percentage of the IFR operations of the nonbased aircraft is similar to that of the based aircraft.

A logistic model is used to establish the relationship between the percentage of itinerant IFR operations and the fleet mix of based aircraft at towered airports. The historical percentages of itinerant IFR operations at towered airports are calculated from the data in the OPSNET. Their corresponding based aircraft information is available in the TAF in terms of five aircraft categories: single-engine aircraft, multiple-engine aircraft, jet-engine aircraft, helicopters, and others. As multiengine aircraft and jet-engine aircraft usually have higher percentage of hours flown under IFR, we used the percentage of based multiengine aircraft $\left(\mathrm{BA}_{M}\right)$ and jetengine aircraft $\left(\mathrm{BA}_{J}\right)$ as the primary determinants of the percentage of IFR operations. The model is given as follows:

$$
\bar{M}_{\mathrm{IFR}}=\frac{\exp \left(E+E_{M} \times \mathrm{BA}_{M}+E_{J} \times \mathrm{BA}_{J}\right)}{\exp \left(E+E_{M} \times \mathrm{BA}_{M}+E_{J} \times \mathrm{BA}_{J}\right)+1},
$$

where $E, E_{M}$, and $E_{J}$ are coefficients to be estimated. Nontowered airports primarily serve GA operations while large and medium hubs mainly serve commercial operations and allow limited GA operations [1]. This suggests that the relationship between the percentage of IFR operations and based aircraft at large and medium hubs may be significantly different from the one at nontowered airports. Therefore, we did not consider large and medium hubs in model calibration 
TABLE 3: Coefficient estimates* from 1998 to 2012.

\begin{tabular}{|c|c|c|c|c|c|c|c|c|c|c|c|c|c|c|c|}
\hline & 1998 & 1999 & 2000 & 2001 & 2002 & 2003 & 2004 & 2005 & 2006 & 2007 & 2008 & 2009 & 2010 & 2011 & 2012 \\
\hline $\begin{array}{l}\text { Number of airports } \\
\text { used in calibration }\end{array}$ & 307 & 315 & 322 & 327 & 332 & 332 & 340 & 343 & 344 & 347 & 347 & 350 & 349 & 351 & 353 \\
\hline E & -1.81 & -1.9 & -1.74 & -1.63 & -1.71 & -1.73 & -1.82 & -1.79 & -1.74 & -1.79 & -1.73 & -1.82 & -1.82 & -1.73 & -1.76 \\
\hline$E_{M}$ & 3.01 & 3.65 & 3.19 & 2.96 & 3.02 & 3.04 & 3.54 & 3.37 & 2.91 & 3.3 & 3.54 & 3.86 & 3.66 & 3.05 & 3.05 \\
\hline$E_{J}$ & 4.8 & 3.99 & 3.89 & 3.57 & 4.11 & 4.34 & 4.26 & 4.22 & 4.29 & 4.45 & 3.36 & 3.28 & 3.45 & 3.46 & 3.64 \\
\hline McFadden's $R^{2}$ & $4.8 \%$ & $5.0 \%$ & $4.4 \%$ & $4.1 \%$ & $4.7 \%$ & $5.2 \%$ & $5.2 \%$ & $5.2 \%$ & $5.0 \%$ & $5.5 \%$ & $4.3 \%$ & $4.6 \%$ & $5.0 \%$ & $4.7 \%$ & $5.0 \%$ \\
\hline
\end{tabular}

${ }^{*}$ Only data at airports other than large and medium hubs are used for model calibration.

TABLE 4: Selected airports for model application.

\begin{tabular}{lccc}
\hline Airport & Location & Category in NIPAS & Itinerant GA operations $^{*}$ \\
\hline William P. Hobby Airport (HOU) & Houston (TX) & Towered medium hub & 53,494 \\
DeKalb Peachtree Airport (PDK) & Atlanta (GA) & Towered GA reliever & 97,988 \\
Destin Airport (DTS) & Okaloosa County (FL) & Nontowered GA & 47,400 \\
McKinnon St. Simons Island Airport (SSI) & St. Simons Island & Nontowered GA & 36,000 \\
\hline
\end{tabular}

${ }^{*}$ Statistics in 2012 from the TAF.

to reduce possible bias. Coefficients are calibrated for each year using corresponding data (data at large and medium hubs are excluded) in TAF (source for explanatory variables) and OPSNET (source for dependent variable).

Table 3 presents the coefficient estimates between 1998 and 2012. All the estimates are significant at $1 \%$ significance level (a 1\% significance level is used throughout this study). Let Odds $\mathrm{IFR}-\mathrm{VFR}_{\mathrm{IFR}}=\bar{M}_{\mathrm{IFR}}$. It is called the odds, which can be considered as a relative probability. Odds IFR-VFR $_{\text {can }}$ be interpreted as the probability of having an IFR itinerant GA operation over that of having a VFR itinerant GA operation at an airport. For the logistic model, $\exp \left(\Delta E_{M}\right)-$ 1 and $\exp \left(\Delta E_{J}\right)-1$ are the change in Odds $s_{\text {IFR-VFR }}$ due to a $\Delta$ change in the variable $\mathrm{BA}_{M}$ and $\mathrm{BA}_{J}$, respectively. It can be observed that based jet-engine aircraft generally contributes more to the percentage of IFR operations than the multiengine aircraft do. For example, in 2012, a $\Delta=10 \%$ increase in the percentage of based jet-engine aircraft would lead to about $44 \%$ increase in the odds of having an IFR operations while the corresponding statistics is about $36 \%$ for multiengine aircraft. However, the coefficient estimates for 2008, 2009, and 2010 seem to suggest otherwise; that is, based multiengine aircraft contribute more to the percentage of IFR operations than the jet-engine aircraft do. The financial crisis in 2008 and the primary usage of aircraft could provide an explanation for this. Based on 2010 GA survey, about $66 \%$ of jet aircraft are primarily used for business and corporate purposes while this number is about $35 \%$ for multiengine aircraft. Therefore, the adverse impact of the financial crisis might be more significant on the jet-engine aircraft.

Note that McFadden's $R^{2}$ of the logistic model is low, which indicates a low goodness-of-fit. The paired dependent $t$-test suggests that a significant difference may exist between the estimated percentage of IFR operations and the observed ones for all the years except 2001, 2002, and 2005. This is consistent with the results reported in Muia and Johnson [11] that the number of based aircraft is not always sufficient to estimate the total number of operations at an airport. Though the target percentages in our model do not need to be errorfree, we believe that, as a further study, more factors should be considered in the logistic model.

\section{Model Application}

4.1. Application to Four Airports. In this part, we applied our model to estimate the percentages of itinerant GA operations by aircraft type and flight rule at four airports shown in Table 4 . They are chosen primarily because (1) they cover both towered and nontowered airports, and commercial service and GA airports; (2) some observed statistics (e.g., ETMS data and airport Master Plan) are available for model validation.

Ideally, to sufficiently capture the situations at an airport, the model should be formulated using information obtained, for example, through airport Master Plan and local airport personnel. However, due to the financial and time limit of this study, the required information is not completely available to the authors, and the model is formulated based on the best of the authors' knowledge.

The Objective Function. For airport HOU and PDK, target percentage $\bar{M}_{\text {IFR }}$ are calculated using their annual counts of IFR operations and total GA operations in the OPSNET (i.e., the former divided by the latter). The ones for airport DTS and SSI are estimated by using the logistics regression model developed in Section 3.2. Table 5 presents the target percentages of IFR itinerant GA operations for the four airports from 1998 to 2012.

To estimate the target percentages in the second and third terms of the objective function, we used the statistics in the GA survey and ETMS sample data. The GA survey is used to estimate hours flown by itinerant GA operations by flight rules. Then, using statistics from ETMS sample data, the hours flown are converted to itinerant GA operations by flight 
TABLE 5: Estimated target percentages of IFR operations $\bar{M}_{\text {IFR }}$ at the four airports.

\begin{tabular}{|c|c|c|c|c|c|c|c|c|c|c|c|c|c|c|c|}
\hline & 1998 & 1999 & 2000 & 2001 & 2002 & 2003 & 2004 & 2005 & 2006 & 2007 & 2008 & 2009 & 2010 & 2011 & 2012 \\
\hline $\mathrm{HOU}^{*}$ & $57.9 \%$ & $57.1 \%$ & $64.6 \%$ & $62.4 \%$ & $65 \%$ & $66.3 \%$ & $70.1 \%$ & $75.1 \%$ & $79.7 \%$ & $77.3 \%$ & $62.9 \%$ & $62.6 \%$ & $71.9 \%$ & $81 \%$ & $83.3 \%$ \\
\hline $\mathrm{PDK}^{*}$ & $52 \%$ & $52.8 \%$ & $52.2 \%$ & $53.5 \%$ & $52 \%$ & $52 \%$ & $53.1 \%$ & $55.4 \%$ & $53.8 \%$ & $51.9 \%$ & $51.1 \%$ & $52.5 \%$ & $50.4 \%$ & $46.6 \%$ & $48.5 \%$ \\
\hline DTS $^{* *}$ & $29.6 \%$ & $32.2 \%$ & $31.4 \%$ & $34 \%$ & $33.9 \%$ & $33.8 \%$ & $34.4 \%$ & $34.9 \%$ & $33.7 \%$ & $34.6 \%$ & $40.7 \%$ & $40.4 \%$ & $40.1 \%$ & $35.3 \%$ & $35.3 \%$ \\
\hline SSI $^{* *}$ & $23.2 \%$ & $22.3 \%$ & $23.9 \%$ & $25 \%$ & $24.1 \%$ & $24 \%$ & $21.6 \%$ & $21.7 \%$ & $21.7 \%$ & $24.6 \%$ & $29.9 \%$ & $25.1 \%$ & $25.6 \%$ & $26 \%$ & $25.7 \%$ \\
\hline
\end{tabular}

${ }^{*}$ Calculated using the airport's statistics in OPSNET from 1998 to $2012 .{ }^{* *}$ Estimated using the logistic regression model with statistics in the TAF from 1998 to 2012 .

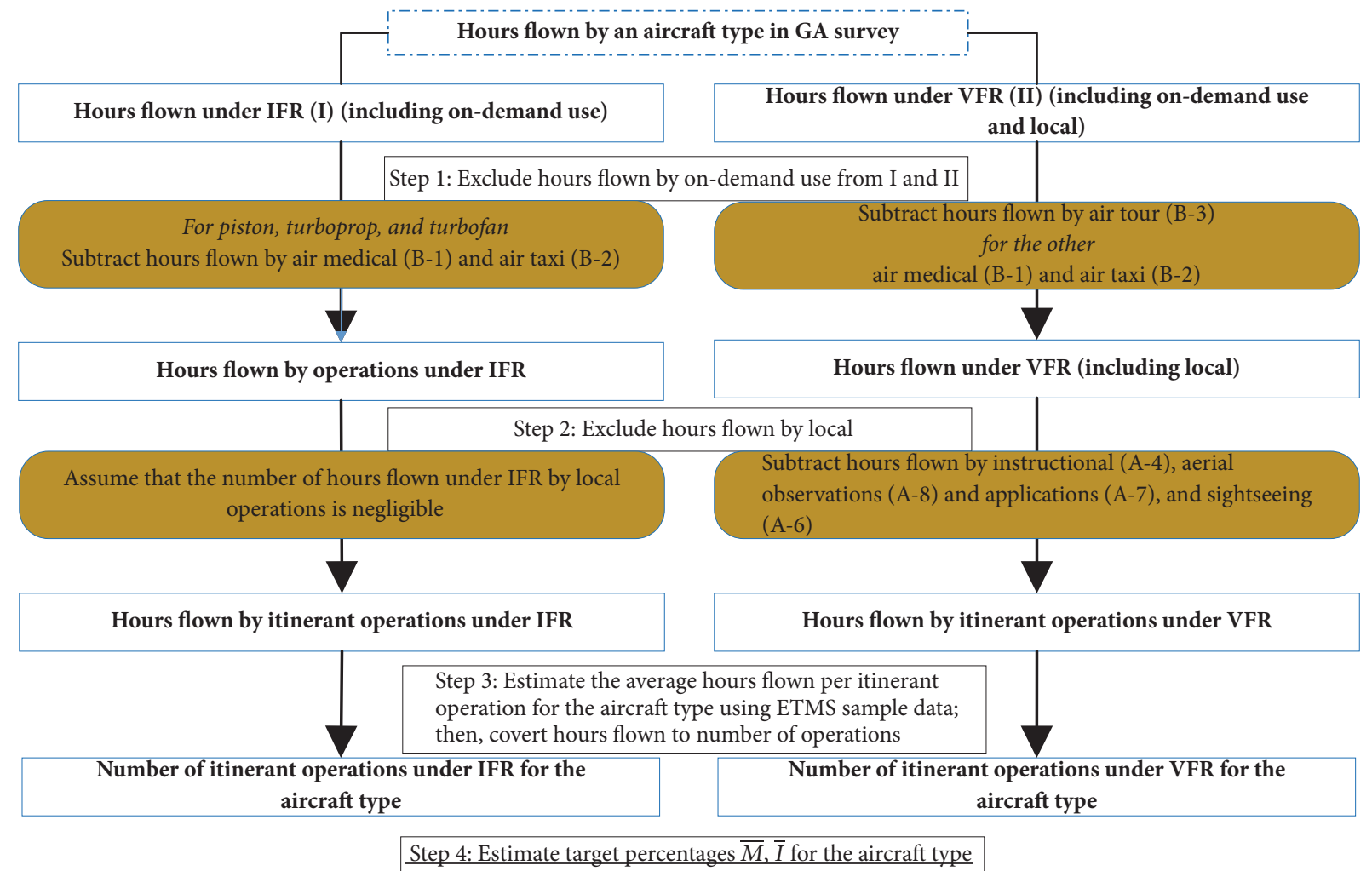

Figure 2: Estimation of target percentages $\bar{M}$ and $\bar{I}$.

rules and used to estimate the target percentages. The detailed process is presented in Figure 2.

As shown in Figure 1, hours flown by an aircraft type under IFR and VFR are generated by both itinerant and local operations for GA and on-demand use. Since only the itinerant GA operations are of interest, we (1) excluded the hours flown by on-demand operations and local operations and (2) converted the hours flown by itinerant GA operations to the number of itinerant GA operations.

To exclude hours flown for on-demand use (Step 1 in Figure 2), hours flown by air tour (B-3 in Figure 1) are assumed to be mainly generated by local operations. They are subtracted from total hours flown under VFR (II in Figure 1) for each aircraft type. We assume that flight operations for the other two purposes (B-1, B-2 in Figure 1) are primarily itinerant under IFR for the first three aircraft types and under VFR for the other aircraft. This assumption is reasonable for turboprop and turbofan since their flight operations are primarily itinerant under IFR. For piston, the hours flown for the two purposes only accounted for about $4 \%$ of its total hours flown. For the other aircraft, about $96 \%$ of the hours flown are under VFR. Therefore, this assumption is not expected to have a significant impact on the estimation results for piston and the other aircraft. For the first three aircraft types, hours flown for the two purposes are subtracted from the total hours flown under IFR (I in Figure 1). For the other aircraft, they are subtracted from the total hours flown under VFR.

To exclude hours flown by local operations for GA use (Step 2 in Figure 2), we assume that hours flown for the purposes of instructional (A-4 in Figure 1), aerial observations (A-8) and applications (A-7), and sightseeing (A-6) are mainly generated by local operations under VFR. These hours are subtracted from the total hours flown under VFR for each aircraft type. 
TABLE 6: Estimated target percentages $\bar{M}, \bar{I}$ at the four airports*.

\begin{tabular}{lccccccc}
\hline Year & & \multicolumn{9}{c}{$1998-2012$} & & \\
& $\bar{M}_{P}$ & $\bar{M}_{B}$ & $\bar{M}_{J}$ & $\bar{I}_{P}$ & $\bar{I}_{B}$ & $\bar{I}_{J}$ & $\bar{I}_{O}$ \\
\hline HOU & & & & & & & \\
PDK & $60.7 \%$ & $8.6 \%$ & $17.6 \%$ & $29.2 \%$ & $83.4 \%$ & $96.9 \%$ & $7 \%$ \\
$\begin{array}{l}\text { DTS } \\
\text { SSI }\end{array}$ & & & & & & & \\
\hline
\end{tabular}

*Estimated using GA survey and ETMS sample data from 2004 to 2012.

To convert hours flown to the number of itinerant operations (Step 3 in Figure 2), we estimated average hours flown per itinerant GA operation for each aircraft type using the ETMS sample data. The estimates are only for operations under IFR as the data set primarily contains IFR operations. We further assume that the average hours flown per itinerant GA operation under VFR is similar to that of IFR. This assumption is reasonable for turbofan as almost all its operations are under IFR. However, the assumption may lead to estimation errors for the other aircraft types as a large portion of their operations are under VFR. The number of itinerant operations by aircraft type and flight rule is estimated by dividing the total hours flown by aircraft type and flight rule by the average hours flown per operation. These numbers are used to calculate $\bar{M}_{P}, \bar{M}_{B}, \bar{M}_{J}, \bar{I}_{P}, \bar{I}_{B}, \bar{I}_{J}$, and $\bar{I}_{O}$ for each aircraft type in each year. However, the ETMS sample data available to this study is from 2004 to 2012. They do not cover all the years listed in Table 5. Therefore, for each target percentage, we use its average from 2004 to 2012 as the target percentage for the four airports and all the years in Table 5. The target percentages in the second and third terms are summarized in Table 6 . The target percentage in the first term is estimated for each airport; therefore, it is reasonable to give it more weight in the objective. We tentatively assigned $\alpha=90 \%, \beta=5 \%$, and $\gamma=5 \%$.

Constraints (4) and Constraints (5) and (6). The three constraints are added for all the four airports.

Constraints (2) and (3) for HOU. In the NIPAS, airport HOU is classified as a medium hub, which serves a large number of commercial jet operations (as many as twice that of GA in 2012 according to the TAF). For GA itinerant operations at this airport, it is reasonable to expect that (1) the percentage of turbofan is high and (2) the percentage of IFR operations is high.

For constraints (2), we require that (1) the percentage of piston operations $\left(M_{P}\right)$ is no more than that of turboprop $\left(M_{B}\right)$; (2) the combination of the percentage of turboprop and piston operations $\left(M_{P}+M_{B}\right)$ is no more than that of turbofan $\left(M_{J}\right)$; (3) the percentage of operations by the other aircraft $\left(M_{O}\right)$ is no more than that of piston $\left(M_{P}\right)$. Constraints (2) are specified as follows:

$$
\begin{aligned}
& M_{P} \leq M_{B}, \\
& M_{O} \leq M_{P}, \\
& M_{P}+M_{B} \leq M_{J} .
\end{aligned}
$$

For constraints (3), we require that (1) the percentage of piston operations under IFR $\left(I_{P}\right)$ is no more than that of turboprop under IFR $\left(I_{B}\right)$; (2) the percentage of turboprop operations under IFR $\left(I_{B}\right)$ is no more than that of turbofan under IFR $\left(I_{J}\right)$; (3) the percentage of operations by the other aircraft under IFR $\left(I_{O}\right)$ is no more than that of piston under IFR $\left(I_{P}\right) ;(4)$ the percentage of turboprop operations under $\operatorname{VFR}\left(1-I_{B}\right)$ is no more than that of turboprop under IFR $\left(I_{B}\right)$; (5) the percentage of turbofan operations under VFR $\left(1-I_{J}\right)$ is no more than that of turbofan under $\operatorname{IFR}\left(I_{J}\right) ;(6)$ the percentage of operations by the other aircraft under IFR $\left(I_{O}\right)$ is no more than that of the other aircraft under VFR $\left(1-I_{O}\right)$. Constraints (3) are specified as follows:

$$
\begin{gathered}
I_{P} \leq I_{B}, \\
I_{B} \leq I_{J}, \\
I_{O} \leq I_{P}, \\
1-I_{B} \leq I_{B}, \\
1-I_{J} \leq I_{J}, \\
I_{O} \leq 1-I_{O} .
\end{gathered}
$$

Constraints (2) and (3) for PDK. Airport PDK has a control tower and is classified as a GA reliever airport for the Atlanta metropolitan area [60]. Therefore, both the number of operations by turbofan and the number of IFR operations are expected to be relatively high at this airport.

For constraints (2), we require that (1) the percentage of turboprop operations $\left(M_{B}\right)$ is no more than that of turbofan $\left(M_{J}\right) ;(2)$ the percentage of operations by the other aircraft $\left(M_{O}\right)$ is no more than that of turboprop $\left(M_{B}\right)$. Constraints (2) are specified as follows:

$$
\begin{aligned}
& M_{B} \leq M_{J}, \\
& M_{O} \leq M_{B} .
\end{aligned}
$$

For Constraints (3), we have the same requirements as those for airport HOU.

Constraints (2) and (3) for DTS and SSI. The two airports are nontowered GA airports that primarily serve local communities. Therefore, the number of turboprop and turbofan operations and the number of IFR operations are expected to be relatively low.

For constraints (2), we require that (1) the percentage of turboprop operations $\left(M_{B}\right)$ is no more than that of piston $\left(M_{P}\right)$; $(2)$ the percentage of turbofan operations $\left(M_{J}\right)$ is no more than that of piston $\left(M_{P}\right)$; (3) the percentage of turbofan operations $\left(M_{J}\right)$ is no more than that of turboprop $\left(M_{B}\right)$; (4) the percentage of turboprop and turbofan operations 
$\left(M_{B}, M_{J}\right)$ is no more than that of the other aircraft $\left(M_{O}\right)$, respectively. Constraints (2) are specified as follows:

$$
\begin{aligned}
& M_{B} \leq M_{P}, \\
& M_{J} \leq M_{P}, \\
& M_{J} \leq M_{B}, \\
& M_{B} \leq M_{O}, \\
& M_{J} \leq M_{O} .
\end{aligned}
$$

For constraints (3), we require that (1) the percentage of piston operations under IFR $\left(I_{P}\right)$ is no more than that of turboprop under IFR $\left(I_{B}\right)$; (2) the percentage of turboprop operations under IFR $\left(I_{B}\right)$ is no more than that of turbofan under IFR $\left(I_{J}\right)$; (3) the percentage of operations by the other aircraft under IFR $\left(I_{O}\right)$ is no more than that of piston under IFR $\left(I_{P}\right)$; $(4)$ the percentage of piston operations under IFR $\left(I_{P}\right)$ is no more than that of piston under VFR $\left(1-I_{P}\right)$; $(5)$ the percentage of turbofan operations under VFR $\left(1-I_{J}\right)$ is no more than that of turbofan operations under IFR $\left(I_{J}\right) ;(6)$ the percentage of operations by the other aircraft under IFR $\left(I_{O}\right)$ is no more than that of the other aircraft under VFR $\left(1-I_{O}\right)$. Constraints (3) are specified as follows:

$$
\begin{gathered}
I_{P} \leq I_{B}, \\
I_{B} \leq I_{J}, \\
I_{O} \leq I_{P}, \\
I_{P} \leq 1-I_{P}, \\
1-I_{J} \leq I_{J}, \\
I_{O} \leq 1-I_{O} .
\end{gathered}
$$

4.2. Results Analysis. Since the majority of IFR GA operations in the ETMS are performed by piston, turboprop, and turbofan, we mainly focused the comparison on the three aircraft types. In our sample, the annual average number of sampled itinerant GA operations by the three aircraft types is 1,774 for HOU, 2,328 for PDK, 831 for DTS, and 379 for SSI.

Table 7 presents the comparison between the estimated percentages and the sampled ones from the ETMS sample. For airport HOU, the paired dependent $t$-test fails to detect any significant differences between the estimated percentages of operations under IFR and the sampled ones for piston and turbofan. However, the estimated percentages of IFR operations for turboprop tend to be slightly higher than the sampled ones. For airport PDK, the estimated percentages of piston operations under IFR are slightly higher than the sampled ones, and those for turboprop and turbofan are slightly lower than the sampled ones. For airports DTS and SSI, the paired dependent $t$-test does not detect any significant differences between the estimated and sampled percentages for the three aircraft types.

We believe that the estimation errors for airports HOU and PDK are possibly due to the following two reasons. First, the specifications of model constraints do not adequately or correctly capture all the situations at the airports. This is mainly due to the fact that necessary information at the two airports is not completely available to our study. Second, it is possible that the operation counts at airports HOU and PDK in the ETMS sample days do not sufficiently represent their operation counts during the year. One of the criteria used to select the sample days from the ETMS is the statistics (e.g., traffic counts) at the core US airports [53, 61]. However, none of the airports are among them, and the sample rate is also relatively low (16 out of 365 , about $5 \%$ ).

Airport DTS's Airport Master Plan [62] contains the number of itinerant GA operations at the airport from 1994 to 2006 in terms of single-engine aircraft, twin-engine aircraft, turboprop, turbofan, and helicopter. The airport was included in the TAF in 1998, and its historical data about its based aircraft is available from this year. Therefore, our comparison is focused on the period of 1998-2006. In addition, its Master Plan provides little information about the definitions of single-engine and twin-engine aircraft. To avoid misunderstanding, we compared the combination of single-engine aircraft, twin-engine aircraft, and helicopter in the airport's Master Plan with the combination of piston and the other aircraft in our model.

Table 8 shows the comparison. Though the paired dependent $t$-test suggests that a significant difference may exist between our estimations and the sampled ones, it can be observed that, from 1998 to 2004, the model estimates for piston and turboprop are relatively close to the sampled ones. Our model underestimates the percentage of turbofan for this period. However, for 2005 and 2006, the estimated percentages of piston operations are about $13 \%$ higher than the sampled ones. The estimated percentages of operations by turboprop and turbofan are about $5 \%$ and $8 \%$ lower than the sampled ones, respectively. It is worthwhile to point out that the sampled percentages in 2005 are significantly different from those in the other years. More specifically, compared with 2004, there is a $9 \%$ drop in the percentage of piston operations and a $5 \%$ increase in the percentages of operations by turboprop and turbofan in 2005. The statistics in the TAF also shows that there is an increase (about a 50\% increase) in the number of based aircraft in this year. These seem to suggest that some significant changes happened at the airport in 2005. These changes might not be sufficiently captured in the model formulation, which could lead to the relatively large estimation errors.

4.3. Application to Airports in the TAF. In this section, we applied the model to the airports in the TAF to estimate the percentages of itinerant GA operations by aircraft type and flight rule. There are 3,335 airports in the TAF and 512 of them are towered airports. For simplicity, we categorized the airports into three groups and used the same model setup for airports in the same group. A summary of the model setups is presented in Table 9. For large and medium hubs (66 towered airports), the model setup is similar to the one for airport HOU. The model setup for the rest of towered airports is similar to the one for airport PDK. For nontowered airports, the model setup is similar to the one for airport DTS and SSI. 
TABLE 7: Observed and estimated percentages of IFR operations at the four airports.

\begin{tabular}{|c|c|c|c|c|c|c|}
\hline \multirow{2}{*}{$\mathrm{HOU}$} & \multicolumn{2}{|c|}{ Piston } & \multicolumn{2}{|c|}{ Turboprop } & \multicolumn{2}{|c|}{ Turbofan } \\
\hline & Observed & Estimated & Observed & Estimated & Observed & Estimated \\
\hline 2004 & $16.56 \%$ & $9.73 \%$ & $18.88 \%$ & $26.92 \%$ & $64.56 \%$ & $63.35 \%$ \\
\hline 2005 & $17.15 \%$ & $10.2 \%$ & $21.54 \%$ & $27.19 \%$ & $61.31 \%$ & $62.61 \%$ \\
\hline 2006 & $11.96 \%$ & $10.63 \%$ & $24.85 \%$ & $27.44 \%$ & $63.2 \%$ & $61.93 \%$ \\
\hline 2007 & $10.14 \%$ & $10.41 \%$ & $23.06 \%$ & $27.31 \%$ & $66.8 \%$ & $62.28 \%$ \\
\hline 2008 & $10.64 \%$ & $9.72 \%$ & $22.86 \%$ & $27.82 \%$ & $66.5 \%$ & $62.46 \%$ \\
\hline 2009 & $11.7 \%$ & $9.77 \%$ & $24.63 \%$ & $27.96 \%$ & $63.67 \%$ & $62.27 \%$ \\
\hline 2010 & $10.78 \%$ & $9.9 \%$ & $25.22 \%$ & $27.02 \%$ & $64 \%$ & $63.08 \%$ \\
\hline 2011 & $8.22 \%$ & $10.76 \%$ & $20.72 \%$ & $27.51 \%$ & $71.06 \%$ & $61.72 \%$ \\
\hline 2012 & $9.5 \%$ & $11.26 \%$ & $21.45 \%$ & $27.8 \%$ & $69.05 \%$ & $60.94 \%$ \\
\hline A significant difference is detected by dependent $t$-test & \multicolumn{2}{|c|}{ No } & \multicolumn{2}{|c|}{ Yes } & \multicolumn{2}{|c|}{ No } \\
\hline \multirow{2}{*}{ PDK } & \multicolumn{2}{|c|}{ Piston } & \multicolumn{2}{|c|}{ Turboprop } & \multicolumn{2}{|c|}{ Turbofan } \\
\hline & Observed & Estimated & Observed & Estimated & Observed & Estimated \\
\hline 2004 & $31.74 \%$ & $37.97 \%$ & $21.44 \%$ & $18.94 \%$ & $46.82 \%$ & $43.09 \%$ \\
\hline 2005 & $35.43 \%$ & $37.23 \%$ & $20.87 \%$ & $19.4 \%$ & $43.7 \%$ & $43.37 \%$ \\
\hline 2006 & $34.4 \%$ & $37.72 \%$ & $20.85 \%$ & $19.09 \%$ & $44.75 \%$ & $43.19 \%$ \\
\hline 2007 & $28.61 \%$ & $38.37 \%$ & $23.84 \%$ & $18.7 \%$ & $47.55 \%$ & $42.93 \%$ \\
\hline 2008 & $32.24 \%$ & $38.63 \%$ & $22.56 \%$ & $18.55 \%$ & $45.2 \%$ & $42.82 \%$ \\
\hline 2009 & $30.42 \%$ & $38.15 \%$ & $24.02 \%$ & $18.83 \%$ & $45.56 \%$ & $43.02 \%$ \\
\hline 2010 & $31.08 \%$ & $38.88 \%$ & $23.64 \%$ & $18.4 \%$ & $45.28 \%$ & $42.72 \%$ \\
\hline 2011 & $27.27 \%$ & $39.73 \%$ & $27.01 \%$ & $19.33 \%$ & $45.73 \%$ & $40.94 \%$ \\
\hline 2012 & $30.23 \%$ & $39.54 \%$ & $21.79 \%$ & $18.09 \%$ & $47.97 \%$ & $42.37 \%$ \\
\hline A significant difference is detected by dependent $t$-test & \multicolumn{2}{|c|}{ Yes } & \multicolumn{2}{|c|}{ Yes } & \multicolumn{2}{|c|}{ Yes } \\
\hline \multirow{2}{*}{ DTS } & \multicolumn{2}{|c|}{ Piston } & \multicolumn{2}{|c|}{ Turboprop } & \multicolumn{2}{|c|}{ Turbofan } \\
\hline & Observed & Estimated & Observed & Estimated & Observed & Estimated \\
\hline 2004 & $46.02 \%$ & $46.56 \%$ & $25.82 \%$ & $24.72 \%$ & $28.16 \%$ & $28.72 \%$ \\
\hline 2005 & $44.64 \%$ & $46.22 \%$ & $26.96 \%$ & $24.87 \%$ & $28.4 \%$ & $28.9 \%$ \\
\hline 2006 & $40.78 \%$ & $46.97 \%$ & $30.29 \%$ & $24.53 \%$ & $28.93 \%$ & $28.51 \%$ \\
\hline 2007 & $47.71 \%$ & $46.42 \%$ & $27.18 \%$ & $24.78 \%$ & $25.1 \%$ & $28.8 \%$ \\
\hline 2008 & $49.65 \%$ & $43.13 \%$ & $28.64 \%$ & $26.32 \%$ & $21.71 \%$ & $30.56 \%$ \\
\hline 2009 & $53.07 \%$ & $43.26 \%$ & $28.5 \%$ & $26.26 \%$ & $18.43 \%$ & $30.49 \%$ \\
\hline 2010 & $53.63 \%$ & $43.41 \%$ & $26.49 \%$ & $26.18 \%$ & $19.87 \%$ & $30.41 \%$ \\
\hline 2011 & $52.88 \%$ & $45.98 \%$ & $25.13 \%$ & $24.99 \%$ & $21.99 \%$ & $29.04 \%$ \\
\hline 2012 & $49.94 \%$ & $45.99 \%$ & $24.63 \%$ & $24.98 \%$ & $25.42 \%$ & $29.03 \%$ \\
\hline A significant difference is detected by dependent $t$-test & \multicolumn{2}{|c|}{ No } & \multicolumn{2}{|c|}{ No } & \multicolumn{2}{|c|}{ No } \\
\hline \multirow{2}{*}{ SSI } & & & Turl & prop & Tur & ofan \\
\hline & Observed & Estimated & Observed & Estimated & Observed & Estimated \\
\hline 2004 & $45.29 \%$ & $57.9 \%$ & $24.85 \%$ & $19.47 \%$ & $29.86 \%$ & $22.63 \%$ \\
\hline 2005 & $57.22 \%$ & $57.73 \%$ & $17.59 \%$ & $19.55 \%$ & $25.19 \%$ & $22.72 \%$ \\
\hline 2006 & $48.55 \%$ & $57.72 \%$ & $20.75 \%$ & $19.55 \%$ & $30.71 \%$ & $22.73 \%$ \\
\hline 2007 & $48.77 \%$ & $54.31 \%$ & $25.06 \%$ & $21.13 \%$ & $26.17 \%$ & $24.56 \%$ \\
\hline 2008 & $44.59 \%$ & $49.64 \%$ & $28.14 \%$ & $23.29 \%$ & $27.27 \%$ & $27.07 \%$ \\
\hline 2009 & $56.98 \%$ & $53.85 \%$ & $19.77 \%$ & $21.34 \%$ & $23.26 \%$ & $24.81 \%$ \\
\hline 2010 & $52.38 \%$ & $53.34 \%$ & $20.24 \%$ & $21.58 \%$ & $27.38 \%$ & $25.09 \%$ \\
\hline 2011 & $42.11 \%$ & $52.91 \%$ & $23.16 \%$ & $21.77 \%$ & $34.74 \%$ & $25.31 \%$ \\
\hline 2012 & $47.69 \%$ & $53.22 \%$ & $20 \%$ & $21.63 \%$ & $32.31 \%$ & $25.15 \%$ \\
\hline A significant difference is detected by dependent $t$-test & & & & & & \\
\hline
\end{tabular}


TABLE 8: Observed and estimated percentages of itinerant operations at airport DTS.

\begin{tabular}{|c|c|c|c|c|c|c|}
\hline \multirow{2}{*}{ DTS } & \multicolumn{2}{|c|}{ Piston } & \multicolumn{2}{|c|}{ Turboprop } & \multicolumn{2}{|c|}{ Turbofan } \\
\hline & Observed & Estimated & Observed & Estimated & Observed & Estimated \\
\hline 1998 & $78.57 \%$ & $83.16 \%$ & $10.5 \%$ & $8.42 \%$ & $10.93 \%$ & $8.42 \%$ \\
\hline 1999 & $79 \%$ & $81.06 \%$ & $10.22 \%$ & $9.47 \%$ & $10.78 \%$ & $9.47 \%$ \\
\hline 2000 & $78.69 \%$ & $81.75 \%$ & $9.91 \%$ & $9.13 \%$ & $11.4 \%$ & $9.13 \%$ \\
\hline 2001 & $76.67 \%$ & $79.62 \%$ & $10.49 \%$ & $10.19 \%$ & $12.84 \%$ & $10.19 \%$ \\
\hline 2002 & $76.16 \%$ & $79.71 \%$ & $10.55 \%$ & $10.15 \%$ & $13.29 \%$ & $10.15 \%$ \\
\hline 2003 & $75.66 \%$ & $79.79 \%$ & $11.44 \%$ & $10.1 \%$ & $12.89 \%$ & $10.1 \%$ \\
\hline 2004 & $73.73 \%$ & $79.37 \%$ & $11.9 \%$ & $10.31 \%$ & $14.37 \%$ & $10.31 \%$ \\
\hline 2005 & $64.7 \%$ & $78.92 \%$ & $16.25 \%$ & $10.54 \%$ & $19.04 \%$ & $10.54 \%$ \\
\hline 2006 & $67.81 \%$ & $79.89 \%$ & $14.44 \%$ & $10.05 \%$ & $17.76 \%$ & $10.05 \%$ \\
\hline A significant difference is detected by dependent $t$-test & \multicolumn{2}{|c|}{ Yes } & \multicolumn{2}{|c|}{ No } & \multicolumn{2}{|c|}{ Yes } \\
\hline
\end{tabular}

TABLE 9: Summary of model setups.

\begin{tabular}{lccc}
\hline Airport type & Large and medium hubs & The other towered airports & Nontowered airports \\
\hline Model setup & Similar to the setup for HOU & Similar to the setup for PDK & Similar to the setup for DTS and \\
$\bar{M}_{\text {IFR }}$ & Calculated using their statistics & Calculated using their statistics & Estimated using the logistic \\
$\bar{M}_{P}, \bar{M}_{B}, \bar{M}_{J}, \bar{I}_{P}, \bar{I}_{B}, \bar{I}_{J}$ & in OPSNET & in OPSNET & regression model \\
Constraints & Same as HOU & Same as PDK & Same as DTS and SSI \\
\hline
\end{tabular}

Similar to the comparison in Table 7, we also compared the estimated percentages of IFR GA operations by piston, turboprop, and turbofan with the observed ones from ETMS sample data. The comparison is focused on 1,953 airports that are in both TAF and sample data. For each of the airports, we compared its estimated percentages of IFR GA operations by the three aircraft types with the sampled ones from 2004 to 2012 with paired dependent $t$-test. For piston, the test does not detect any significant differences between the model estimates and the sampled ones at 938 airports (about $48 \%$ of the airports in the comparison). The corresponding statistics for turboprop and turbofan are 1,140 airports (about 58.3\%) and 789 airports (about 40.4\%), respectively.

The TAF contains historical annual total number of itinerant GA operations at airports. With estimated percentages of itinerant GA operations by turbofan at TAF airports, we could estimate the annual total number of itinerant GA operations by turbofan at TAF airports. The TAF covers almost all the airports that could support the operations of turbofan aircraft. Therefore, the annual total number of itinerant GA operations by turbofan at US airports could be estimated by summing up the corresponding statistics at each of TAF airports.

The FAA provides business jet activities in its Business Jet Report $[5,51]$. The statistics in the report are derived from flight database in the ETMS. In Table 10, we compared the annual total number of itinerant GA operations by turbofan at US airports from our model estimates with the business jet activities from the FAA's report. The comparison suggests that our estimates are generally lower. One possible explanation for this is that some business jet activities in FAA's report are not for GA purposes. For example, we investigated the
TABLE 10: Comparison of itinerant GA operations by turbofan at US airports with business jet activities.

\begin{tabular}{cccc}
\hline Year & $\begin{array}{c}\text { Statistics from } \\
\text { FAA's report }\end{array}$ & $\begin{array}{c}\text { Total number from } \\
\text { model estimates }\end{array}$ & Error \\
\hline 2001 & $3,826,564$ & $4,017,607$ & $4.99 \%$ \\
2002 & $4,198,012$ & $3,625,190$ & $-13.65 \%$ \\
2003 & $4,285,420$ & $3,497,194$ & $-18.39 \%$ \\
2004 & $4,606,122$ & $3,414,436$ & $-25.87 \%$ \\
2005 & $4,727,826$ & $3,457,646$ & $-26.87 \%$ \\
2006 & $4,745,746$ & $3,213,646$ & -32.284 \\
2007 & $4,824,960$ & $3,279,068$ & $-32.04 \%$ \\
2008 & $4,291,104$ & $3,469,796$ & $-19.14 \%$ \\
2009 & $3,449,204$ & $3,181,971$ & $-7.75 \%$ \\
2010 & $3,842,314$ & $3,002,607$ & $-21.85 \%$ \\
2011 & $3,955,400$ & $3,116,261$ & $-21.22 \%$ \\
2012 & $3,982,236$ & $3,007,729$ & $-24.47 \%$ \\
\hline
\end{tabular}

activities of three typical business jets in FAA's report: C56X, C560, and H25B. In the ETMS sample data for 2012, about $40 \%, 80 \%$, and $80 \%$ of their operations are classified as GA. The rest of their operations are classified as commercial and air taxi, which are not GA.

\section{Summary and Conclusion}

Historical airport-level GA activity plays an important role in many airport managerial and operational decision-making 




Figure 3: Model summary.

processes. Detailed historical data would be important in making appropriate decisions.

In this study, we presented an optimization model to estimate historical airport-level itinerant GA operations by four aircraft types and two flight rules. A flowchart that provides a summary of the model and its application is given in Figure 3. This study is a contribution to the literature in that it provides researchers and practitioners with a method to estimate airport-level itinerant GA operations in more detail. We would like to point out a specific application of our method. The FAA has been collecting aeronautical data on public-use airports in the US via Form 5010 [63] and making the data available to the general public. The number of itinerant GA operations is one of the statistics that the FAA collects. The number could be collected by local airports using the methods introduced in Section 1. These numbers are used in the TAF as the itinerant GA operations for nontowered airports [6]. However, the number being reported is the combination of all aircraft types and flight rules. Using our method, local airports or the FAA could provide more detailed estimates for the general public by separating the total number of itinerant operations into the number of itinerant operations by aircraft type and flight rule.

The model in this study belongs to the type of estimation methods that use posterior observations. In general, they are less expensive than deploying traffic counters at airports to directly count the operations year-around. For the comparison of the model in this study with the existing methods of the same type, we would like to point out the following. First, the existing methods mainly estimate the total number of operations (i.e., the combination of aircraft type and flight rule) at an airport. In contrast, the method in this study estimates the percentages of itinerant GA operations by aircraft type and flight rule at an airport. By applying the percentage estimates to the estimate of total number, the number of GA operations by aircraft type and flight rule at an airport could be estimated. From this point of view, the method in this study is complementary to the existing methods. Second, since the model in this study estimates GA operations in more detail, intuitively the accuracy of its estimation may be lower than some existing methods. Muia and Johnson [11] studied the estimation accuracy (in terms of total number) of three statistical estimation methods with data from 16 small, towered airports. They concluded that statistical extrapolation using two-week sample counts per season generates the best estimates with the estimation errors ranging from $-11.6 \%$ to $15.8 \%$. We investigated the estimation accuracy of our model at four airports, which cover both towered and nontowered airports. The results show that model estimates are generally consistent with observations. Third, for some posterior observations, it could be expensive or time-consuming to collect on a large-scale basis. For example, using statistical extrapolation requires sample traffic counts over a period (e.g., two weeks per season), which is usually collected by using traffic counters. If the cost of using traffic counters is high, it could be prohibitively expensive to apply the method to a large number of airports. In contrast, we have shown that it is possible to apply the method in this study to a large number of airports (e.g., all the airports in the TAF) without a significant cost.

Lastly, we summarize several limitations of this study and potential future work. The target percentages are introduced to keep percentage estimates within reasonable ranges. They should vary among airports and years. However, due to data unavailability, target percentages $\bar{M}$ and $\bar{I}$ (given in Table 6) are estimated using statistics from the GA survey and the ETMS sample at national level. They are assumed to be the same for all airports for all years. In addition, a logistic regression model is developed to estimate target percentage $\bar{M}_{\text {IFR }}$ for nontowered airports. The model's goodness-of-fit 
is low. We performed a sensitivity analysis of estimation accuracy with respect to $\bar{M}_{\text {IFR }}$. Different error levels (e.g., $\pm 5 \%, \pm 20 \%$, and $\pm 50 \%$ ) are introduced into the $\bar{M}_{\mathrm{IFR}} \mathrm{s}$ used in Section 4.3, and the comparison between the estimation results and the statistics from the ETMS sample at 1,953 airports is repeated for each error level. We observed that the estimation accuracy for turbofan shows relatively low sensitivity while the estimation accuracy for piston and turboprop deteriorates as the underestimation error increases. A possible future work is to consider more explanatory variables to improve the goodness-of-fit and hence reduce estimation error. Furthermore, a sensitivity analysis for the other two target percentages is needed to determine their impact on estimation accuracy. It is also important to clarify that this paper only provides a way to estimate the target percentages at an airport. Other methods (e.g., a survey of pilots at an airport) could also be used to estimate the percentages. The estimation results are validated on four airports with the data from ETMS sample and airport Master Plan. We suggest that the validation be performed on more airports. Since the cost of collecting very accurate historical data at airport level could be high, it is also important that collection/estimation methods are cost-effective [64]. Therefore, studies that evaluate and compare existing collecting/estimation methods in terms of cost, accuracy, and efficiency should be developed to, for example, select cost-effective options for airports with different needs and constraints.

\section{Disclosure}

The views and models presented in this paper are the authors' sole responsibility.

\section{Conflicts of Interest}

The authors declare that there are no conflicts of interest regarding the publication of this paper.

\section{Acknowledgments}

This study is partially supported by the FAA under Grant [FAA AWA DTFAWA-11-D-00017]. The authors would like to thank David Chin, Thea Graham, and Dan Murphy at the FAA ATO office.

\section{References}

[1] Federal Aviation Administration, "National Plan of Integrated Airport Systems (NPIAS) 2011-2015," Tech. Rep., Federal Aviation Administration, Washington, DC, USA, 2010.

[2] T. Zakaria, "Estimation of aircraft operations at nontowered airports in the Delaware valley region," Transportation Research Record: Journal of the Transportation Research Board, vol. 1158, pp. 37-46, 1988.

[3] M. J. Muia, "An analysis of the methods used to calculate customer operations at non-towered airports and of the associated managerial uses of operations information," Tech. Rep., The Union Institute Graduate College, Cincinnati, Ohio, USA, 2000.
[4] Federal Aviation Administration, "Appendix A: Methodology for the 2010 General Aviation and Part 135 Acitivity Survey," 2010.

[5] Federal Aviation Administration, "Conducting Unmanned Aircraft Flight Operations Under Federal Aviation Administration Regulations," 2014, http://aspmhelp.faa.gov/index.php/ Operations_Network_\%28OPSNET\%29.

[6] Federal Aviation Administration, "Terminal Area Forecast Summary Fiscal Years 2011-2040,” 2010.

[7] GRA Inc, "Forecasting aviation activity by airport," Tech. Rep., GRA, Inc, Jenkintown, Pa, USA, 2001.

[8] Federal Aviation Administration, "Airport Master Plans," 2007, Advisory Circular AC 150/5070-6B.

[9] Center for Transportation Research and Education, "Acceptable method of counting aircraft operations at non-tower airports," Tech. Rep., Center for Transportation Research and Education, Iowa State University, Ames, Iowa, USA, 1996.

[10] M. J. Muia, "Counting aircraft operations at non-towered airports: a synthesis of airport practice," ACRP Report 129, Transportation Research Board, Washington, DC, USA.

[11] M. J. Muia and M. E. Johnson, "Evaluating methods for counting aircraft operations at non-towered airports," ACRP Program Synthesis 4, Transportation Research Board, Washington, DC, USA, 2015.

[12] M. Hoekstra, "Model for estimating general aviation operations at non-towered airports," Tech. Rep., Federal Aviation Administration, Washington, DC, USA, 2000.

[13] GRA Inc, "Model for estimating general aviation operations at non-towered airports using towered and non-towered airport data," Tech. Rep., GRA, Inc, Jenkintown, Pa, USA, 2001.

[14] R. Black and J. R. Chimka, "Re-estimating and remodeling general aviation operations," International Journal of Applied Aviation Studies, vol. 11, no. 1, pp. 47-56, 2011.

[15] M. Johnson and Y. Gu, "Estimating airport operations at general aviation airports using the FAA NPIAS airport categories," International Journal of Aviation, Aeronautics, and Aerospace, vol. 4, no. 1, pp. 339-354, 2017.

[16] A. Ghobrial, "A model to forecast aircraft operations at general aviation airports," Journal of Advanced Transportation, vol. 31, no. 3, pp. 311-323, 1997.

[17] L. Dou, L. David, J. Jesse, and K. Peter, "A small aircraft transportation system (sats) demand model," Tech. Rep., Logistics Management Institute, Mclean, Va, USA, 2001.

[18] T. Li and A. A. Trani, "A model to forecast airport-level General Aviation demand," Journal of Air Transport Management, vol. 40, pp. 192-206, 2014.

[19] T. Li and A. A. Trani, "A procedure to estimate the airport-level market share of itinerant GA operations by aircraft type," in Proceedings of the 16th Integrated Communications, Navigation, and Surveillance Conference, ICNS 2016, Herndon, Va, USA, 2016.

[20] M. L. Ford and R. Shirack, "Estimating aircraft activity at nontowered airports: results of the aircraft activity counter demonstration project," Transportation Research Record, pp. 2429, 1984.

[21] Lousiana Department of Transportation and Development, "Lousiana airports activity study: summary report," Tech. Rep., Lousiana Department of Transportation and Development, Baton Rouge, La, USA, 1985.

[22] R. Milone and K. Flick, "General aviation activity model," Tech. Rep., Metropolitan Washington Council of Governments, Washington, DC, USA, 1995. 
[23] Texas Department of Transportation, "Aircraft activity counter report," Tech. Rep., Aviation Division,Texas Department of Transportation, Austin, Tex, USA, 1994.

[24] T. Li, H. Baik, and A. A. Trani, "A method to estimate the historical US air travel demand," Journal of Advanced Transportation, vol. 47, no. 3, pp. 249-265, 2013.

[25] T. Li and H. Baik, "Addressing two issues in the airline origin and destination survey," in Proceedings of the Transportation Research Board 92nd Annual Meeting, Washington, DC, USA, 2013.

[26] T. Li, "A bi-level model to estimate the US air travel demand," Asia-Pacific Journal of Operational Research, vol. 32, no. 2, Article ID 1550009, 1550009 pages, 2015.

[27] T. Li, H. Baik, and T. Spencer, "An optimization model to estimate the air travel demand for the United States," in Proceedings of the 14th Integrated Communications, Navigation and Surveillance Conference, ICNS 2014, pp. E21-E211, Herndon, Va, USA, 2014.

[28] T. Li, "A demand estimator based on a nested logit model," Transportation Science, 2016.

[29] M. J. Maher, "Inferences on trip matrices from observations on link volumes: a Bayesian statistical approach," Transportation Research. Part B. Methodological, vol. 17, no. 6, pp. 435-447, 1983.

[30] E. Cascetta, "Estimation of trip matrices from traffic counts and survey data: a generalized least squares estimator," Transportation Research Part B: Methodological, vol. 18, no. 4-5, pp. 289299, 1984.

[31] M. G. Bell, "The estimation of origin-destination matrices by constrained generalised least squares," Transportation Research. Part B. Methodological, vol. 25, no. 1, pp. 13-22, 1991.

[32] H. Yang, Q. Meng, and M. G. H. Bell, "Simultaneous estimation of the origin-destination matrices and travel-cost coefficient for congested networks in a stochastic user equilibrium," Transportation Science, vol. 35, no. 2, pp. 107-123, 2001.

[33] E. Codina and J. Barceló, "Adjustment of O-D trip matrices from observed volumes: an algorithmic approach based on conjugate directions," European Journal of Operational Research, vol. 155, no. 3, pp. 535-557, 2004.

[34] P. Chootinan, A. Chen, and W. Recker, "Improved path flow estimator for origin-destination trip tables," Transportation Research Record, vol. 1923, no. 1, pp. 9-17, 2005.

[35] J. Doblas and F. G. Benitez, "An approach to estimating and updating origin-destination matrices based upon traffic counts preserving the prior structure of a survey matrix," Transportation Research Part B: Methodological, vol. 39, no. 7, pp. 565-591, 2005.

[36] Y. Nie, H. M. Zhang, and W. W. Recker, "Inferring origindestination trip matrices with a decoupled GLS path flow estimator," Transportation Research Part B: Methodological, vol. 39, no. 6, pp. 497-518, 2005.

[37] J. T. Lundgren and A. Peterson, "A heuristic for the bilevel origin-destination-matrix estimation problem," Transportation Research Part B: Methodological, vol. 42, no. 4, pp. 339-354, 2008.

[38] A. Chen, S. Ryu, and P. Chootinan, " $L_{\infty}$-Norm path flow estimator for handling traffic count inconsistencies: Formulation and solution algorithm," Journal of Transportation Engineering, vol. 136, no. 6, Article ID 008006QTE, pp. 565-575, 2010.

[39] E. Fernandes and R. R. Pacheco, "Efficient use of airport capacity," Transportation Research Part A: Policy and Practice, vol. 36, no. 3, pp. 225-238, 2002.
[40] M. Janic, "Modelling the capacity of closely-spaced parallel runways using innovative approach procedures," Transportation Research Part C: Emerging Technologies, vol. 16, no. 6, pp. 704730, 2008.

[41] B. Mirkovic, "Airport apron capacity estimation-model enhancement," Procedia-Social and Behavioral Sciences, vol. 20, pp. 1108-1117, 2011, (The State of the Art in the European Quantitative Oriented Transportation and Logistics Research 14th Euro Working Group on Transportation \& 26th Mini Euro Conference; 1st European Scientific Conference on Air Transport).

[42] G. Andreatta, P. Dell'Olmo, and G. Lulli, "An aggregate stochastic programming model for air traffic flow management," European Journal of Operational Research, vol. 215, no. 3, pp. 697-704, 2011.

[43] C. N. Glover and M. O. Ball, "Stochastic optimization models for ground delay program planning with equity-efficiency tradeoffs," Transportation Research Part C: Emerging Technologies, vol. 33, pp. 196-202, 2013.

[44] A. D’Ariano, D. Pacciarelli, M. Pistelli, and M. Pranzo, "Realtime scheduling of aircraft arrivals and departures in a terminal maneuvering area," Networks, vol. 65, no. 3, pp. 212-227, 2015.

[45] M. Samà, A. D’Ariano, P. D’Ariano, and D. Pacciarelli, "Air traffic optimization models for aircraft delay and travel time minimization in terminal control areas," Public Transport, vol. 7, no. 3, pp. 321-337, 2015.

[46] M. Samà, A. D’Ariano, P. D’Ariano, and D. Pacciarelli, "Scheduling models for optimal aircraft traffic control at busy airports: tardiness, priorities, equity and violations considerations," Omega, vol. 67, pp. 81-98, 2017.

[47] R. Rylander and M. Björkman, "Annoyance by aircraft noise around small airports," Journal of Sound and Vibration, vol. 205, no. 4, pp. 533-537, 1997.

[48] Federal Aviation Administration, "Environmental Desk Reference for Airport Actions," 2007.

[49] Federal Aviation Administration, "FAA aerospace forecasts fiscal years 2013-2033," Tech. Rep., Federal Aviation Administration, Washington, DC, USA.

[50] T. Li and A. A. Trani, "Modeling the impact of fuel price on the utilization of piston engine aircraft," in Proceedings of the 2013 13th Annual Integrated Communications, Navigation and Surveillance Conference, ICNS 2013, Herndon, Va, USA, 2013.

[51] Federal Aviation Administration, "Traffic Flow Management System (TFMS)," 2014, http://aspmhelp.faa.gov/index.php/ Traffic_Flow_Management_System_(TFMS).

[52] C. G. Rodarte, Knowledge Management in the Enhanced Traffic Management System [Master's, thesis], Massachusetts Institute of Technology, Cambridge, Mass, USA, 1974.

[53] F. Cheng, J. Gulding, B. Baszczewski, and R. Galaviz, "An optimization model for sample day selection in NAS-wide modeling studies," in Proceedings of the 11th Integrated Communications, Navigation and Surveillance Conference: Renovating the Global Air Transportation System, ICNS 2011, pp. L61-L68, Herndon, Va, USA, 2011.

[54] H. Baik, T. Li, and N. K. Chintapudi, "Estimation of flight delay costs for U.S. domestic air passengers," Transportation Research Record, vol. 2177, pp. 49-59, 2010.

[55] Federal Aviation Administration, "Air Traffic Organization Policy: Facility Statistical Data, Reports, and Forms," 2008, N JO 7210.695. 
[56] S. William and G. Richard, "ACRP program synthesis 2: airport aviation activity forecasting," Tech. Rep., GRA, Inc., Jenkintown, Pa, USA, 2007.

[57] R. W. Pike, Optimization for Engineering Systems, Van Nostrand Reinhold, New York, NY, USA, 1986.

[58] M. Avriel, Nonlinear Programming: Analysis and Methods, Prentice-Hall, Inc, Englewood Cliffs, NJ, USA, 1976.

[59] A. D. Waren and L. S. Lasdon, "The status of nonlinear programming software," Operations Research. The Journal of the Operations Research Society of America, vol. 27, no. 3, pp. 431456, 1979.

[60] Dekalb Peachtree Airport, "Dekalb Peachtree Airport," 2017, https://www.dekalbcountyga.gov/airport.

[61] F. Cheng, J. Gulding, B. Baszczewski, R. Galaviz-Schomisch, and A. Chow, "Modeling the effect of weather conditions in sample day selection using an optimization method," in Proceedings of the 2012 12th Integrated Communications, Navigation and Surveillance Conference: Bridging CNS and ATM, ICNS 2012, pp. N31-N311, Herndon, Va, USA, 2012.

[62] Destin Airport, "Destin-fort walton beach airport: airport master plan update," Tech. Rep., Destin-Fort Walton Beach Airport, Eglin AFB, Fla, USA, 2010.

[63] Federal Aviation Administration, "Form 5010-1 - Airport Master Record (Existing Public Use Airports)," 2003, https:// www.faa.gov/forms/index.cfm/go/document.information/documentID/185474.

[64] Airport Cooperative Research Program, "FY 2017 Research Problem Statements 17-03-06: Accurate Measurement of Airport Operations Counts," 2017, http://onlinepubs.trb.org/ onlinepubs/acrp/fy2017ps/17-03-06French.pdf. 


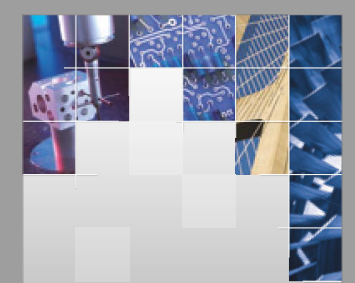

\section{Enfincering}
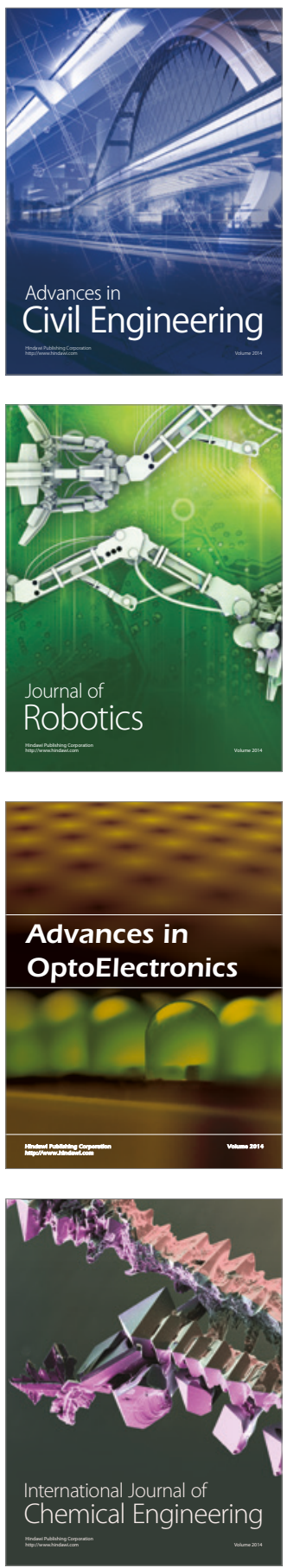



The Scientific World Journal

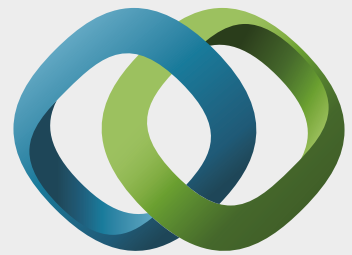

\section{Hindawi}

Submit your manuscripts at

https://www.hindawi.com
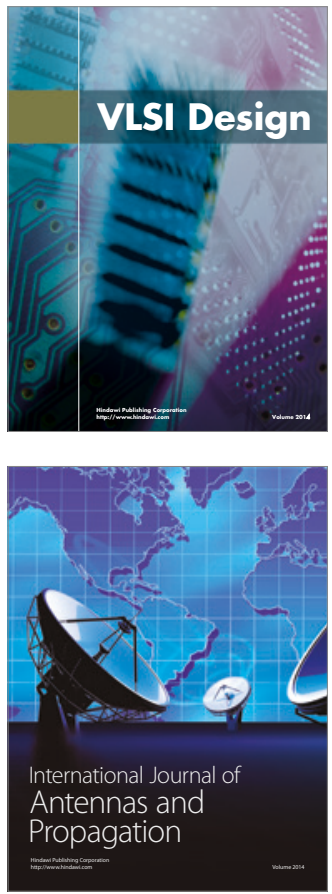

\section{Rotating}

Machinery

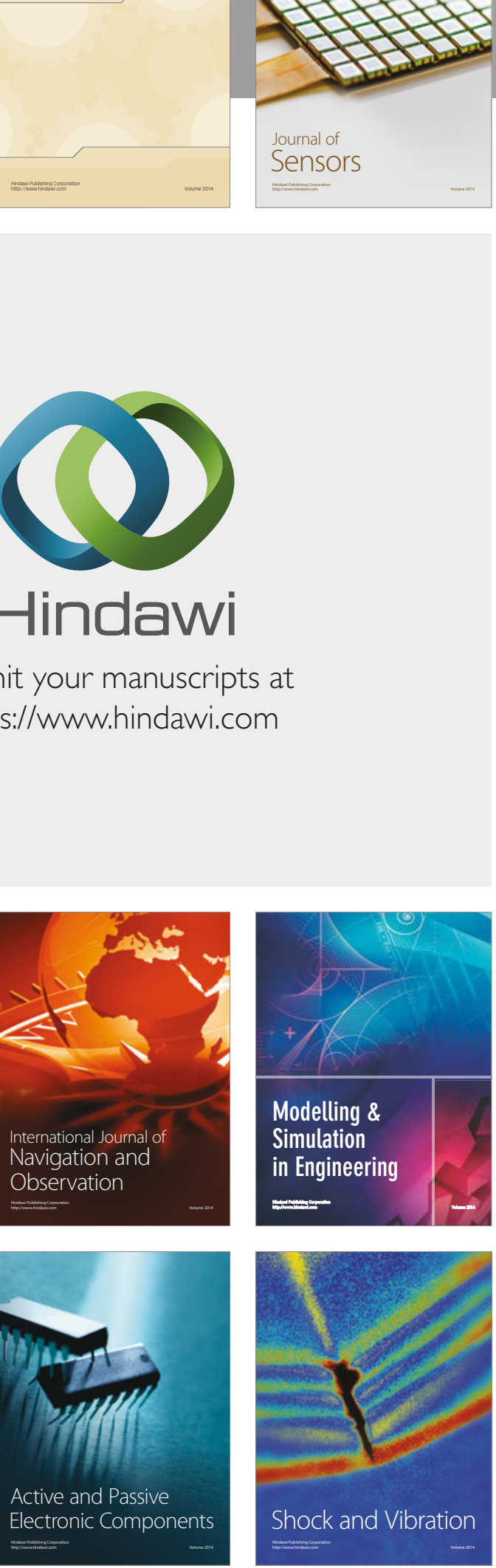
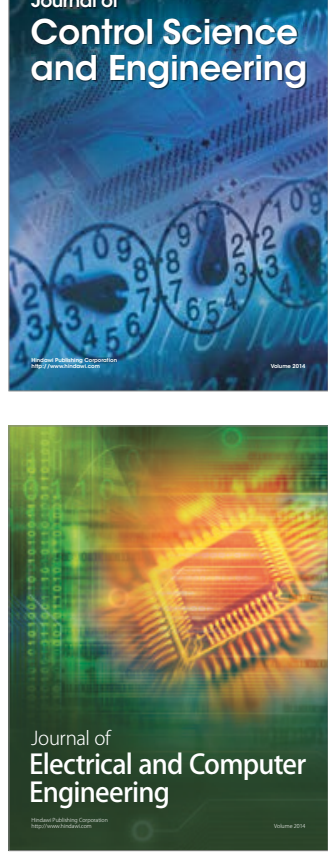

Distributed

Journal of

Control Science

and Engineering
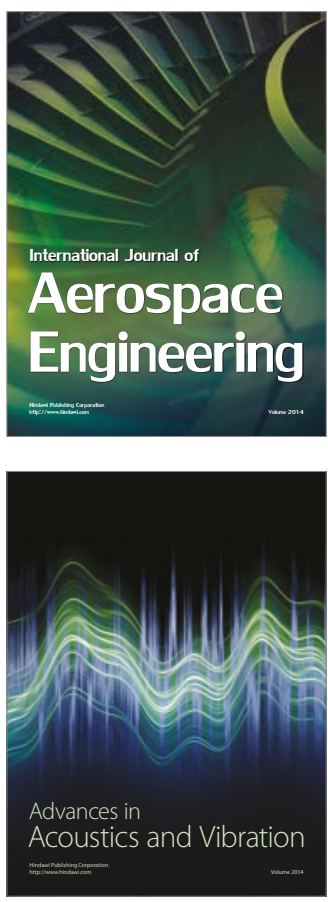

Sensor Networks 